\title{
LOW COST X BAND RADAR SYSTEM FOR MULTIPLE TARGET DETECTION
}

\author{
Amit Singh ${ }^{1}$, Abhishek Thakur ${ }^{2}$, Ankur Taparia ${ }^{3}$ \\ ${ }^{I}$ B.Tech Final Year, Electrical Engineering Department, Delhi Technological University, Delhi, India \\ ${ }^{2}$ B.Tech Final Year, Electrical Engineering Department, Delhi Technological University, Delhi, India \\ ${ }^{3}$ B.Tech Final Year, Electrical Engineering Department, Delhi Technological University, Delhi, India
}

\begin{abstract}
Radar based object detection systems have been in place for about a century serving various purposes like detection of ships aircrafts, spacecraft, navigation, reading weather formations and terrain mapping. With the advent of better packing technologies and signal conditioning circuits radars have become compact and affordable thus offering comparatively low cost solutions than other systems based on imaging. This paper describes an X band Radar System for multiple target detection that can work as an effective solution for obstacle detection and collision avoidance system to be used in automated braking systems. This radar system is capable of detecting multiple targets from the clutter encountered by the radar signals within a range of 20 metres. The paper discusses various moving target indicator techniques employed for multiple target detection and speed of the object in real time. This is particularly useful in automotive industries where the low cost motion sensors are needed in variety of applications which are necessary to ensure the safety of the passengers. To ensure a good signal to noise ratio we have used non coherent integration so that the radar signal is recognizable among the clutter. Moving Target Indicator techniques are used primarily to detect the number of objects in the range of the radar. This is carried out along with adaptive constant false alarm thresholding technique so that the object can be detected among the clutter and noise accurately. An Arduino Uno is used for detecting the objects and speed after processing on the non-coherently integrated pulse.
\end{abstract}

Keywords: Radar, Clutter. CFAR, MTI

\section{INTRODUCTION}

The term RADAR, introduced as an acronym for Radio Detection And Ranging is now a specially coined term referring to systems employing radio waves for object and target detection. Radar detects objects using radio waves to determine the range, altitude, direction, or speed of objects. Radars have wide-ranging applications such as detecting aircrafts, ships, space crafts, guided missiles, motor vehicles and mapping terrain. The radar transmitter transmits radio waves through the radar transmitting antenna which are electromagnetic in nature. These waves travel through the medium to the object incurring losses on the way. The object, generally metal interacts with the radio waves producing electromagnetic field of its own so that no net electric field is formed inside the metal. This electromagnetic field that is produced to oppose the incident electromagnetic field then travels to the receiver antenna.

The existing implementations employing HB 100 Doppler radars have developed systems that are capable of detecting single targets using continuous wave operation ${ }^{[1]}$. This paper discusses Doppler radar for detecting multiple targets using Moving Target Indicator techniques in pulse Doppler mode.

\subsection{Received Energy of $X$ band Signals}

Radar systems are classified on the basis of frequency of operation in $\mathrm{S}$ band $(3 \mathrm{GHz}), \mathrm{C}$ band $(5 \mathrm{GHz}), \mathrm{X}$ band $(10$ $\mathrm{GHz}$ ), and other higher frequency bands. $\mathrm{X}$ band radars are imaging radars that offer a comparatively reliable low cost solution than other motion sensors. The received signal energy from the radar is given by the equation:

$$
\begin{gathered}
\text { Transmit Transmit Spread Losses Target Spread Receive Dwell } \\
\text { Power Gain Factor } \begin{array}{l}
\text { RCS Factor Aperture Time } \\
\text { Received Signal } \\
\text { Energy }
\end{array}=\left(P_{T}\right)\left(\frac{4 \pi A}{\lambda^{2}}\right)\left(\frac{1}{4 \pi R^{2}}\right)\left(\frac{1}{L}\right)(\sigma)\left(\frac{1}{4 \pi R^{2}}\right)(A)(\tau)
\end{gathered}
$$

Fig 1: Received Signal Energy Formula ${ }^{[2]}$

This received energy at the receiver is mixed with noise. Noise are of following types galactic, solar, manmade interference noise, atmospheric, ground, transmitter, receiver, waveguide and duplexer noise.

\subsection{Detection of Radar Pulses in Noise}

The detection of radar pulses involves two parameters namely probability of detection and probability of false alarm. On a fixed threshold, higher the Signal to Noise Ratio higher would be the probability of detection. The Signal to Noise Ratio of Radar is given by following equation.

$$
\left.\mathrm{SNR}=10 \log _{10} \text { [signal power/noise power }\right]
$$




$\begin{array}{ll}\begin{array}{l}\text { Signal Power reflected } \\ \text { from target and received } \\ \text { by radar }\end{array} & \mathrm{Pr}_{\mathbf{r}}=\frac{\mathrm{P}_{\mathrm{t}} \mathrm{t}}{4 \pi \mathrm{R}^{2}} \frac{\sigma \mathrm{Ae}}{4 \pi \mathrm{R}^{2}} \\ \text { Average Noise Power } & \mathrm{N}=\mathrm{kT}_{\mathrm{s}} \mathrm{B}_{\mathrm{n}} \\ \text { Signal to Noise Ratio } & \frac{\mathrm{S}}{\mathrm{N}}=\frac{\mathrm{P}_{\mathrm{r}}}{\mathrm{N}}\end{array}$

Fig 2: Signal Power and Avg. Noise Power Formulas ${ }^{[2]}$

$$
\frac{S}{N}=\frac{P_{t} G^{2} \lambda^{2} \sigma}{(4 \pi)^{3} R^{4} K \text { Ts } B_{n} L}
$$

Fig 3: Signal to Noise Ratio Formula ${ }^{[2]}$

-The System Noise Temperature Ts, is divided into 3 components :

$$
\mathrm{Ts}=\mathrm{Ta}+\operatorname{Tr}+\mathrm{LrTe}
$$

-Ta is the contribution from the antenna

- Apparent temperature of sky (from graph)

- Loss within antenna

- $\mathrm{Tr}$ is the contribution from the RF components between the antenna and the receiver

- Tempe`rature of RF components

- $\mathrm{Lr}$ is the loss of input RF components

-Te is the temperature of the receiver

- Noise factor of receiver

The detection of target echoes in noise involves integration of pulses, fluctuating target issues and adaptive thresholding techniques. The integration of pulses can be done using coherent and non-coherent techniques. Coherent integration involves addition of in phase and quadrature quantities of complex radar return signal. These voltages are then computed, averaged and matched with a threshold. In general non-coherent integration, the pulse magnitude is calculated which is then averaged so that it can be compared with a threshold. In coherent techniques no information is lost while in non-coherent techniques phase information is lost. Coherent techniques are more efficient than noncoherent integration techniques.

\section{MULTIPLE TARGET DETECTION USING MTI TECHNIQUES}

Targets can be distinguished from the clutter using Moving Target Indicator technique and Pulse Doppler technique. Moving target Indicator techniques use low pulse repetition frequency and short waveforms to separate targets and clutter. Pulse Doppler technique classifies targets into different velocity regimes providing velocity data along with separation of targets from clutter. This technique uses long waveforms for their operation.

A two pulse MTI canceller can be used to illustrate the principal of multiple object detection. The amplitude of return signal from the moving objects changes in consecutive pulses owing to the Doppler shift while the fixed clutter returns fixed echoes. The subtraction of subsequent pulses eliminates the clutter from the return signal and gives the multiple moving objects at different range cells. The following block diagram depicts method deployed for multiple target detection.

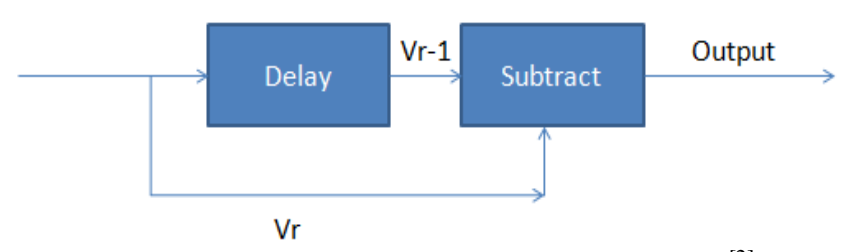

Fig 4: 2 Pulse MTI Canceller Block Diagram ${ }^{[2]}$

For 2 pulse MTI canceller,

$$
\mathbf{V}_{\text {output }}=\mathbf{V}_{\mathrm{i}+1}-\mathbf{V}_{\mathbf{i}}
$$

For 3 pulse MTI canceller,

$$
V_{\text {output }}=V_{i}-2 V_{i-1}+V_{i-2}
$$

\subsection{Adaptive Thresholding Technique for Radar} Signals

Constant false alarm rate (CFAR) thresholding can be used to adaptively threshold the radar signals. For adaptive thresholding the noise floor of the non-coherently integrated signal should be computed using adaptive thresholding. In mean level CFAR technique we process the range cells in window slides. The cell under test is matched to a threshold using mean value of other range cells in the window. This process can detect false targets in sharp clutters and interference. To avoid this Greatest of Mean level CFAR technique is used in which the surrounding range cells are divided into left and right halves which are then averaged. The greatest noise level range half is used to determine the threshold.

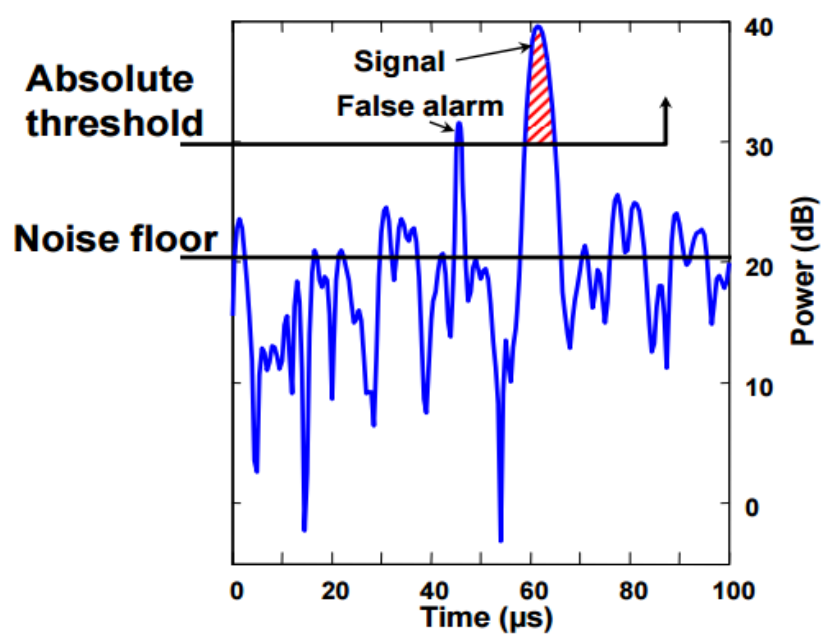

Fig 5: Disadvantage of Absolute Thresholding Technique ${ }^{[2]}$ 


\section{PRACTICAL IMPLEMENTATION}

The radar system is designed using the $\mathrm{HB}-100^{[3]}$ pulsed microwave Doppler sensor module. The range of the module is up to 20 meters which is enough for automatic braking systems. Doppler shift output is observed from IF terminal when movement is detected in the field of detection. The magnitude of the Doppler Shift is in proportions to reflection of transmitted energy and is in microvolts. A high gain low frequency bandwidth amplifier is connected to the IF pin to amplify the Doppler shift to a level that can be read by development platform like Arduino Uno. Frequency of Doppler shift is calculated using the algorithm to determine the velocity of targets. Doppler frequency is related to velocity of motion through:

$$
\mathbf{F}_{\mathbf{d}}=2 \mathrm{~V}\left[\frac{\mathrm{F}_{\mathrm{t}}}{\mathrm{C}}\right] \cos \theta
$$

Fig 6: Doppler Frequency Formula ${ }^{[3]}$

Where

$F_{d}=$ Doppler Frequency

$\mathrm{V}=$ Velocity of the target

$\mathrm{F}_{\mathrm{t}}=$ Transmit frequency

$\mathrm{C}=$ Speed of light $\left(3 \times 10^{8} \mathrm{~m} / \mathrm{sec}\right)$

$\Theta=$ the angle between the target moving direction and the axis of the module.

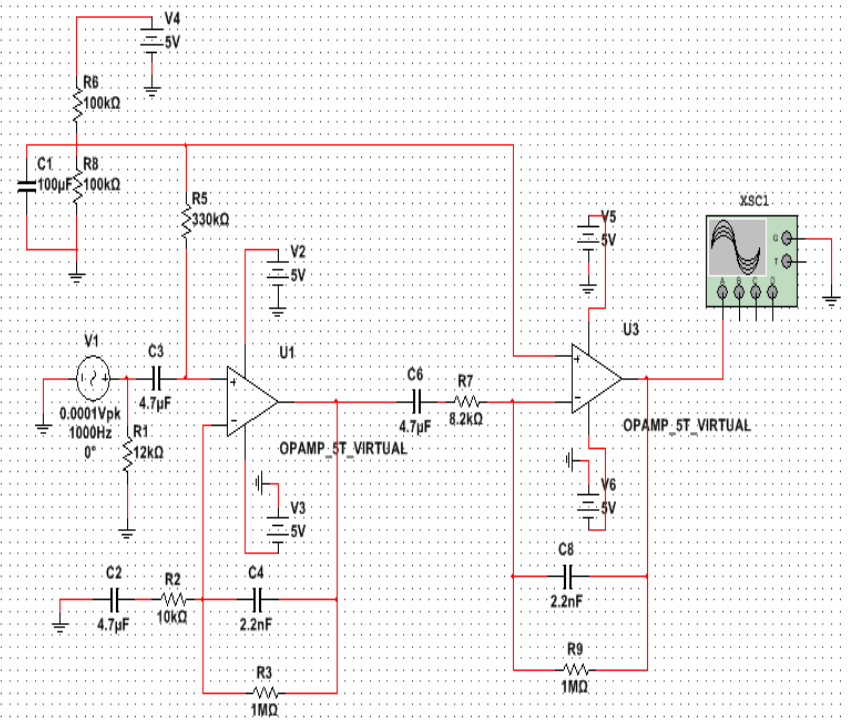

Fig 7: Radar Amplifier Circuit Diagram

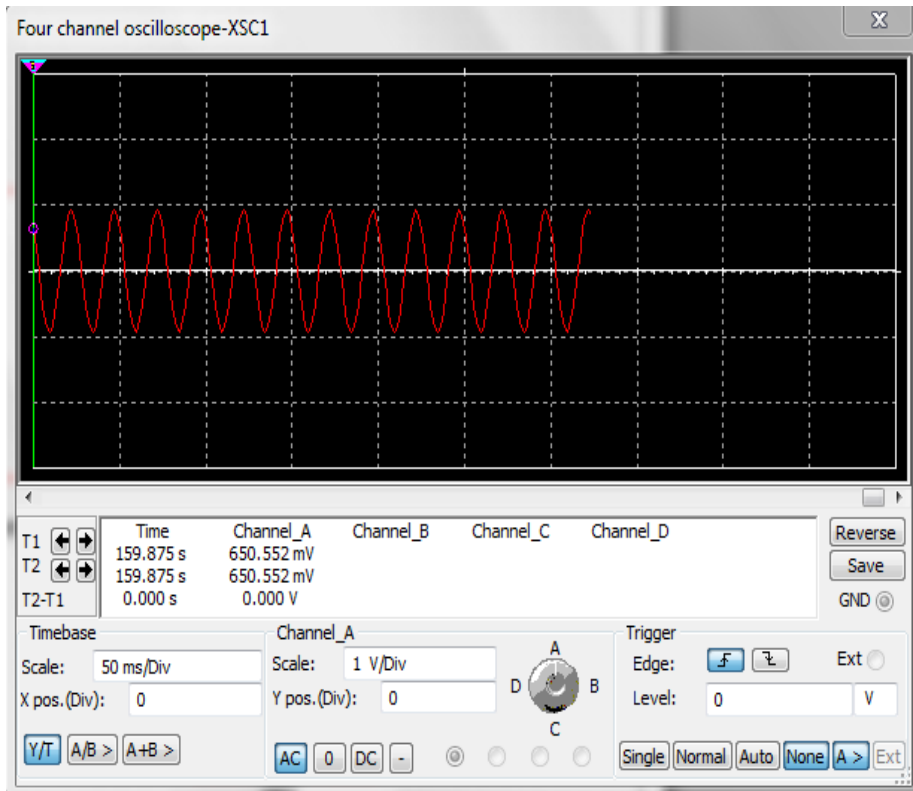

Fig 8: Simulation Of Amplifier Circuit in MultiSim

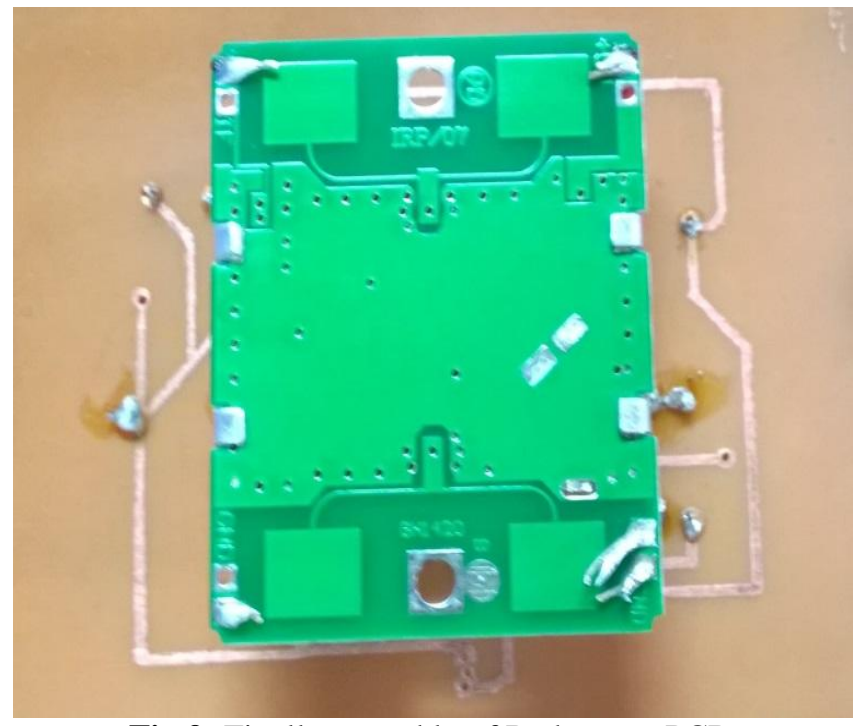

Fig 9: Finally assembly of Radar on a PCB

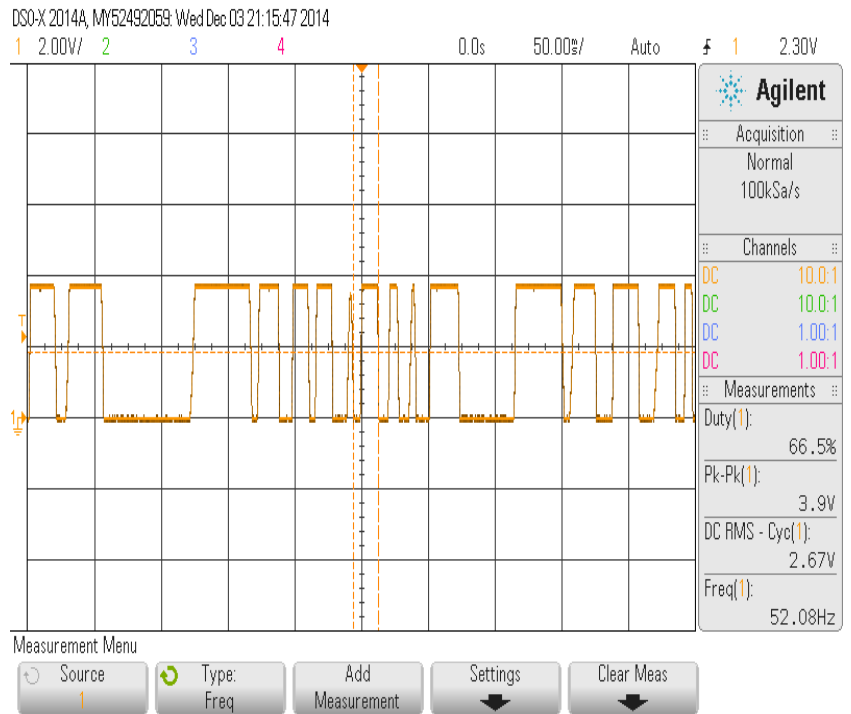

Fig 10: Output of the Amplifier Stage 


\subsection{Algorithm}

The Arduino Uno is initialized with Pin 11 as Pulse generator. A PWM of $2 \mathrm{kHz}$ pulse repetition frequency is generated with a pulse width of 10 microseconds high time using the delay Microseconds() function and giving 240 microseconds low time for the digital pin. The pulse received is converted from analog to digital and this is sent to non-coherent integrator wherein the consecutive pulses are simply added and averaged to increase SNR. These integrated pulses are then differenced from subsequent pulses to get the number of objects. The objects in the path are detected using Greatest of Mean level CFAR technique adaptive thresholding with the Arduino storing the range gates in an array and then thresholding each range point with the greater of the mean of the next and previous ten range points. The non-coherently integrated pulses are passed through a zero crossing detector ${ }^{[4]}$ or comparator to get a square wave. The frequency counter library of Arduino is then used to compute the frequency and hence the speed.

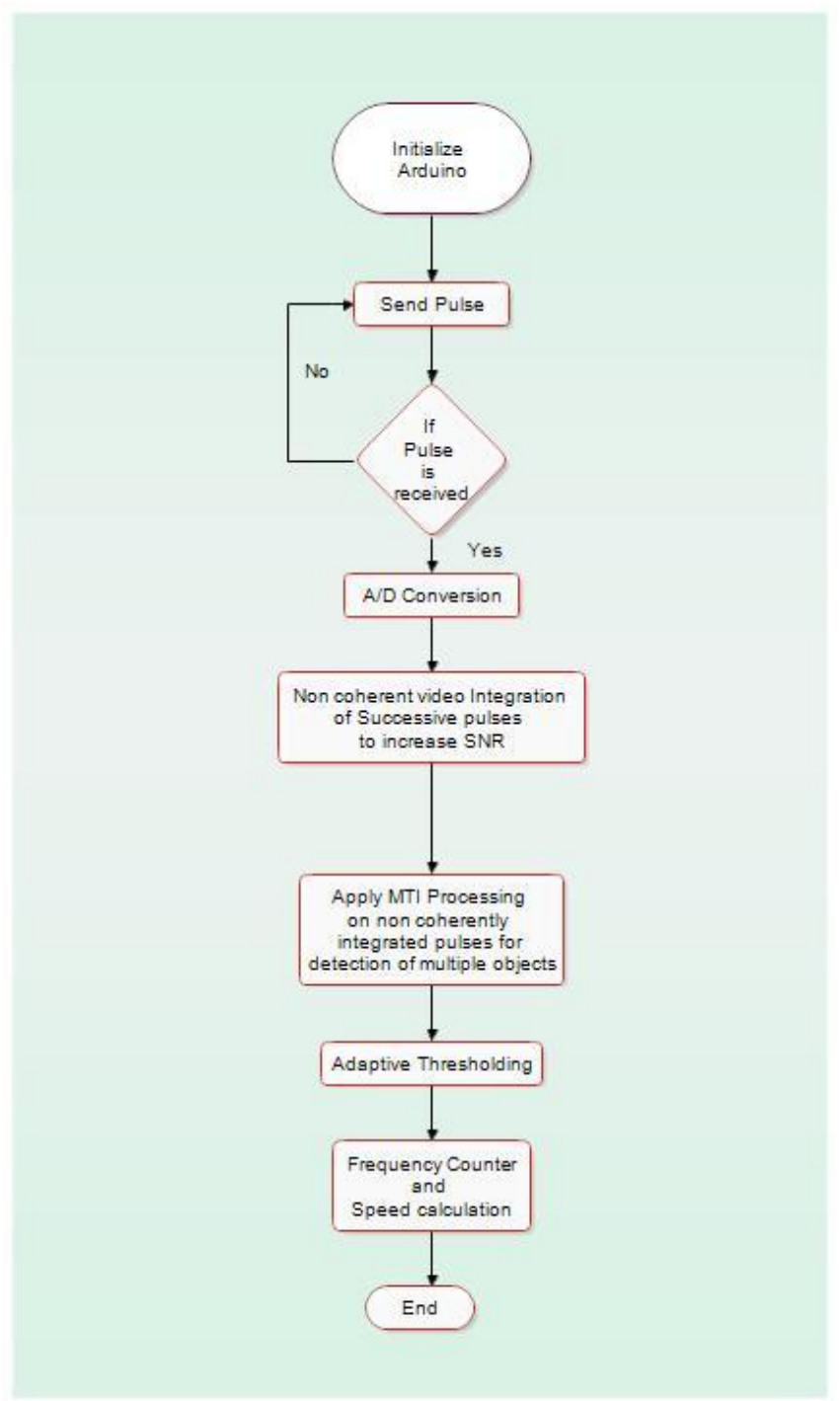

Fig 11: Flowchart of the whole Algorithm

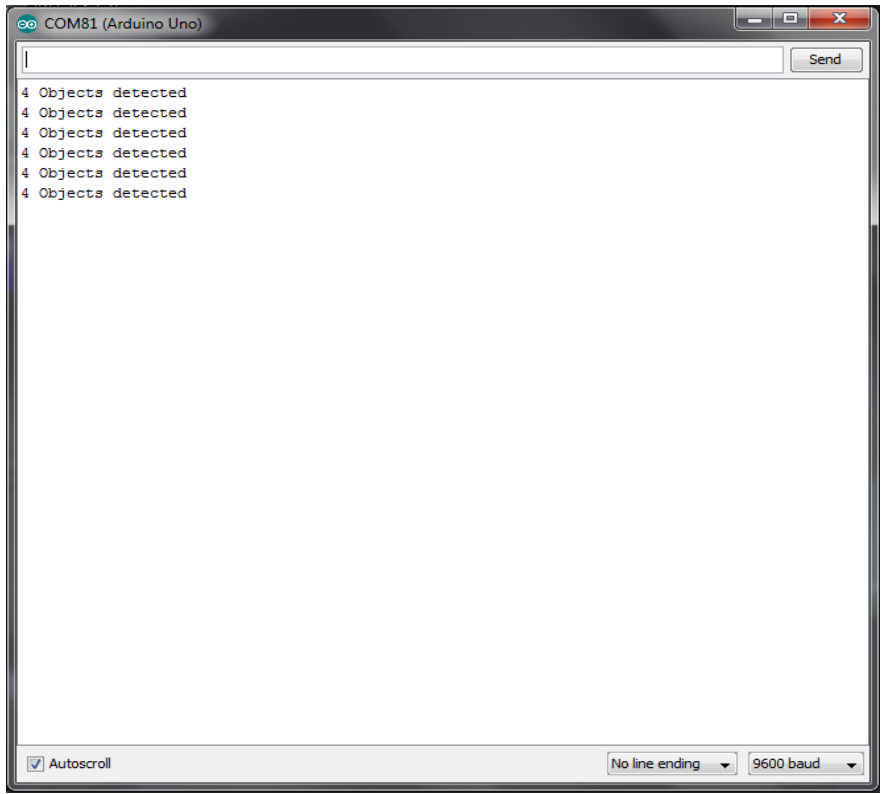

Fig 12: Arduino Object Detection Output

\section{CONCLUSION}

The radar system is promising but constitutes a lot of irregularities and clutter which can be eliminated using Doppler filter banks. So instead of MTI processing, pulse Doppler filter techniques would be our future research work.

As the automotive industry is flourishing day by day, we need more and more cost effective solutions to accommodate all economic classes of the society. The cutting edge technologies of the generation are necessary.

Table -1: Test results of radar system

\begin{tabular}{|l|l|l|}
\hline S.No & Doppler Frequency $(\mathbf{H z})$ & Speed $(\mathbf{k m} / \mathbf{h r})$ \\
\hline 1. & 20.0 & 1.026 \\
\hline 2. & 52.08 & 2.672 \\
\hline 3. & 66.2 & 3.396 \\
\hline 4. & 101.53 & 5.209 \\
\hline 5. & 305.78 & 15.688 \\
\hline
\end{tabular}

\section{ACKNOWLEDGEMENTS}

We acknowledge the support and guidance of Mr. Ram Bhagat, Assistant Professor, Electrical Engineering, Delhi Technological University.

\section{REFERENCES}

[1]. Mohammed Altawim, Ahmed Alahmadi , Mohammed Bonais , Ben Soh , Fahad Algarni (2013),"Radar Vehicle Detector Mote",[Online] http://www.erpublications.com/uploaded_files/download/do wnload_06_04_2013_01_12_49.pdf

[2]. "Introduction to Radar Systems", https://www.ll.mit.edu/workshops/education/videocourses/in troradar/lecture1/lecture.pdf 
[3]. Agilsense, "HB100 Microwave Sensor Application Note", Agilsense

[4]. Asha G Hagargund, Udayshankar R, Rashmi.N (2013),'Radar based cost effective vehicle speed detection using zero cross detection",[Online] http://iraj.in/journal/journal_file/journal_pdf/1-9-

139029869701-05.pdf

[5]. Skolnik, M., Introduction to Radar Systems, New York, McGraw-Hill, 3rd Edition, 2001

[6]. Barton, D. K., Modern radar System Analysis, Norwood, Mass., Artech House, 1988

\section{BIOGRAPHIES}

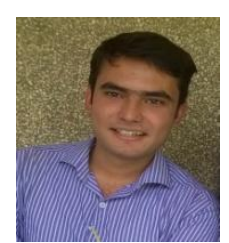

Amit Singh is a final year student of Bachelor of Technology in Electrical Engineering at Delhi Technological University. He has interests in image processing, electronic design and embedded systems. He loves innovating new products and developing ideas that make life simple

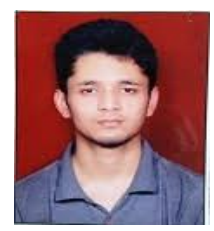

Abhishek Thakur is a student of B.Tech in Delhi Technological University. He is an electrical engineer with interest in electronics and embedded systems. He did his schooling from Lakshmipat Singhania School. He loves being around hardware engineering and likes reading in his past time

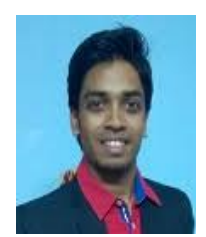

Ankur Taparia is pursuing Bachelor of Technology in Electrical Engineering from Delhi Technological University. His education is from Vivekanand School, Delhi. His main interest is in electronics especially in integrated circuits and loves to work on hardware part. He has contributed a lot in this research and looking forward to realize many more things regarding this with his colleagues. He is a very ethical, hardworking person and loves playing sports a lot. 